\title{
An enhanced computational platform for investigating the roles of regulatory RNA and for identifying functional RNA motifs
}

\author{
Tzu-Hao Chang ${ }^{1}$, Hsi-Yuan Huang ${ }^{2}$, Justin Bo-Kai Hsu², Shun-Long Weng 2,3,4,5,6, Jorng-Tzong Horng ${ }^{7,8,9^{*}}$, \\ Hsien-Da Huang ${ }^{2,3^{*}}$
}

From The Eleventh Asia Pacific Bioinformatics Conference (APBC 2013)

Vancouver, Canada. 21-24 January 2013

\begin{abstract}
Background: Functional RNA molecules participate in numerous biological processes, ranging from gene regulation to protein synthesis. Analysis of functional RNA motifs and elements in RNA sequences can obtain useful information for deciphering RNA regulatory mechanisms. Our previous work, RegRNA, is widely used in the identification of regulatory motifs, and this work extends it by incorporating more comprehensive and updated data sources and analytical approaches into a new platform.

Methods and results: An integrated web-based system, RegRNA 2.0, has been developed for comprehensively identifying the functional RNA motifs and sites in an input RNA sequence. Numerous data sources and analytical approaches are integrated, and several types of functional RNA motifs and sites can be identified by RegRNA 2.0: (i) splicing donor/acceptor sites; (ii) splicing regulatory motifs; (iii) polyadenylation sites; (iv) ribosome binding sites; (v) rhoindependent terminator; (vi) motifs in mRNA 5'-untranslated region (5'UTR) and 3'UTR; (vii) AU-rich elements; (viii) C-to-U editing sites; (ix) riboswitches; $(x)$ RNA cis-regulatory elements; (xi) transcriptional regulatory motifs; (xii) user-defined motifs; (xiii) similar functional RNA sequences; (xiv) microRNA target sites; (xv) non-coding RNA hybridization sites; (xvi) long stems; (xvii) open reading frames; (xviii) related information of an RNA sequence. User can submit an RNA sequence and obtain the predictive results through RegRNA 2.0 web page.
\end{abstract}

Conclusions: RegRNA 2.0 is an easy to use web server for identifying regulatory RNA motifs and functional sites. Through its integrated user-friendly interface, user is capable of using various analytical approaches and observing results with graphical visualization conveniently. RegRNA 2.0 is now available at http://regrna2.mbc.nctu.edu.tw.

\section{Background}

Numerous functional RNA motifs have been identified as playing significant roles in many essential biological processes, including transcriptional and post-transcriptional regulation of gene expression, control of mRNA stability, alternative splicing, and transcription termination. The biological activities of functional RNA motifs usually rely

\footnotetext{
* Correspondence: horng@db.csie.ncu.edu.tw; bryan@mail.nctu.edu.tw ${ }^{2}$ Institute of Bioinformatics and Systems Biology, National Chiao Tung University, Hsin-Chu 300, Taiwan

${ }^{7}$ Department of Computer Science and Information Engineering, National Central University, Chung-Li 320, Taiwan Full list of author information is available at the end of the article
}

on a combination of their primary sequences and specific secondary structures, which act as target sites of RNAbinding factors or directly interact with translation machinery [1]. For instance, riboswitches are metabolitebinding domain within a specific mRNA, and can regulate both transcription and translation by binding their corresponding targets $[2,3]$.

Several databases were established for collecting functional RNA molecules [1,4-14]. UTRdb [1] is a database of 5 ' and 3' untranslated sequences of eukaryotic mRNAs. It provides specialized information including the presence of nucleotide sequence patterns already demonstrated by experimental analysis to have some functional roles, and
C Biomed Central 
these patterns have been collected into the UTRsite database. Rfam [4,5] is a database comprehensively collecting families of non-coding RNA (ncRNA) genes as well as cisregulatory RNA elements. Each family is represented by a multiple sequence alignment of known and predicted representative members, and annotated with a consensus base-paired secondary structure. It facilitates the identification and classification of new members of known RNA families, and provides the glimpses of conservation of multiple ncRNA families across a wide taxonomic range. fRNAdb $[6,7]$ is a database hosting a large collection of ncRNA sequence data from public non-coding databases, and provides related annotations, such as sequence ontology classification and source organisms. AEdb is a database for alternative exons and their properties from numerous species, and it forms the manually curated component of alternative splicing database (ASD) [8]. The data in AEdb is gathered from literature where these exons have been experimentally verified. The adenylate uridylate-rich elements (AREs or AU-rich element) mediate the rapid turnover of mRNA encoding proteins that regulate cellular growth and body response to exogenous agent such as microbes and environmental stimuli. ARED $[9,10]$ is a human AU-rich element-containing mRNA database. A 13-bp ARE pattern was computationally derived using MEME, and five clusters were generated from ARE sequences. NONCODE $[11,12]$ is an integrated knowledge database designed for analysis of ncRNAs. All ncRNAs in NONCODE were confirmed by consulting the references manually and more than $80 \%$ data are from experiments. microRNAs (miRNAs) are small RNA molecules, which are $\sim 22 \mathrm{nt}$ sequences, and participate in gene post-transcriptional regulation and degradation of mRNA by hybridizing to miRNA target sites. miRBase [13] is the central online repository for miRNA nomenclature, sequence data annotation and target prediction. It provides a range of data to facilitate studies of miRNA genomics. TRANSFAC [14] is a knowledge-base containing published data on eukaryotic transcription factors, their experimentallyproven binding site, and regulated genes.

Various approaches were developed for identifying functional RNA motifs or elements [15-26]. GeneSplicer [15] was developed for detecting splice sites in eukaryotic mRNA by combining several techniques, such as maximal dependence decomposition (MDD) and Markov model, that have already proven successful in characterizing the patterns around the donor and acceptor sites. polya_svm [16] was developed for predicting mRNA polyadenylation site using a Support Vector Machine (SVM) featuring 15 over-represented cis-regulatory elements in various regions surrounding. RBSfinder [17] is a probabilistic method to improve the accuracy of gene identification systems at finding precise translation start sites. TransTermHP [18] can rapidly and accurately detecting rho-independent transcription terminators. CURE [19] was developed for predicting C-to-U RNA editing site in plant mitochondria by incorporating both evolutionary and biochemical information. miRanda [20] was developed for finding genomic targets for miRNAs. RiboSW [21] is a systematic method for identifying 12 kinds of riboswitches based on RNA conserved functional sequences and conformations. PatSearch [22] was developed for searching specific combinations of oligonucleotide consensus sequences, secondary structure motifs and position-weight matrices (PWMs). ERPIN [23] is a practical approach for the automatic derivation of an RNA signature from a sequence alignment and secondary structure, and finding the occurrence in sequence databases. Several profiles have been constructed to search any input sequence for the presence of some RNA genes and elements on ERPIN web server. INFERNAL [24] is an implementation of a general stochastic context-free grammars (SCFG) based approach for RNA database searches and multiple alignment. It is used to annotate RNAs in genomes in conjunction with the Rfam families by covariance models, a special case of SCFGs designed for modeling RNA consensus sequence and structure. MATCH [25] is an approach for searching transcription factor binding sites with specific position-weight matrices (PWM). RNAMotif [26] is an RNA secondary structure definition and search algorithm, and commonly used for searching user-defined RNA motifs.

Analysis of functional RNA motifs and sites in RNA sequences can obtain useful information for deciphering RNA regulatory mechanisms. Our previous work, RegRNA [27], is widely used to identify regulatory motifs and miRNA target sites, and has been cited 50 times. However, various types of functional RNA motifs and identification approaches were continuously accumulated and developed in recent years. In order to comprehensively identify functional RNA motifs, a more complete and updated analysis platform is crucial.

This work presents an integrated web server, RegRNA 2.0 , for identifying functional RNA motifs and sites in an input RNA sequence. Numerous data sources, such as Rfam [4], fRNAdb [6] and UTRsite [1], and identification approaches, such as GeneSplicer [15], RiboSW [21] and RBSfinder [17], were integrated in RegRNA 2.0, and other additional information, such as GC-content ratio and RNA accessibility, are also presented on the web page. User can submit an RNA sequence through our user-friendly interface, and obtain the predictive results with graphical visualization.

\section{Methods}

\section{Data collection}

The functional RNA motifs and sites supported in RegRNA 2.0 are categorized into several types: (i) splicing 
donor/acceptor sites; (ii) splicing regulatory motifs; (iii) polyadenylation sites; (iv) ribosome binding sites; (v) rho-independent terminator; (vi) motifs in mRNA 5'-untranslated region (5'UTR) and 3'-UTR; (vii) AU-rich elements; (viii) C-to-U editing sites; (ix) riboswitches; (x) RNA cis-regulatory elements; (xi) transcriptional regulatory motifs; (xii) user-defined motifs; (xiii) similar functional RNA sequences; (xiv) microRNA target sites; (xv) non-coding RNA hybridization sites; (xvi) long stems; (xvii) open reading frames; (xviii) related information of an RNA sequence.
The process flow of RegRNA 2.0 is depicted in Figure 1. Numerous functional RNA motifs and sites were collected from a variety of databases and websites including Rfam [4], ERPIN [23], RiboSW [21], UTRsite [1], AEdb [8], ARED [10], fRNAdb [7], NONCODE [11], miRBase [28] and TRANSFAC [14]. As shown in Table 1, different prediction models, sequences and patterns are incorporated into RegRNA 2.0. There are 209 covariance models (CMs) of Rfam cis-regulatory families, 11 profiles of ERPIN RNA elements, 12 descriptors of RiboSW riboswitches, 48 models of UTRsite motifs and 2,171 transcription factor

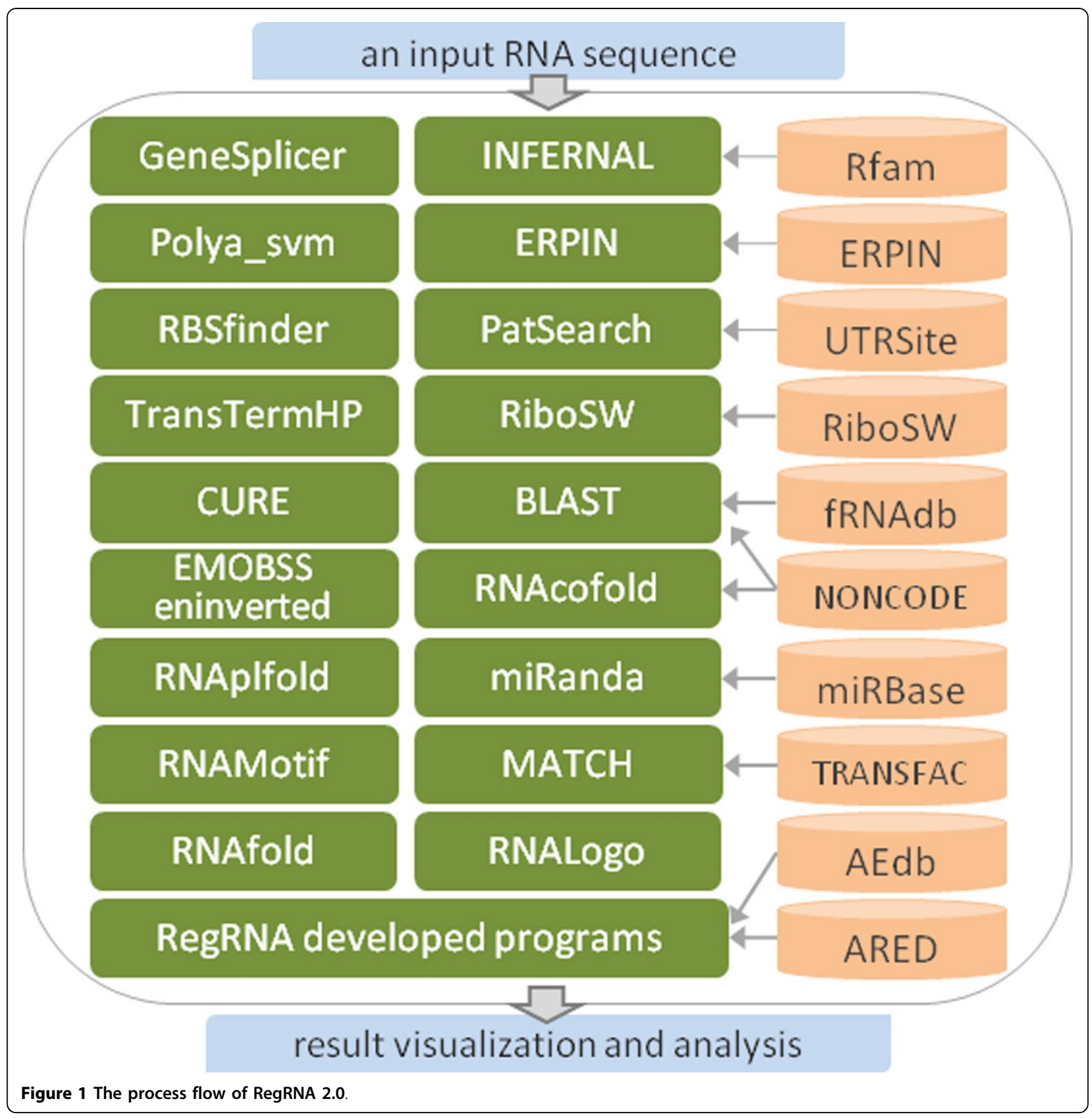


Table 1 Statistics of types of functional RNA motifs supported in RegRNA 2.0

\begin{tabular}{|c|c|c|c|}
\hline Types of functional RNA motifs & Incorporated data & Incorporated approaches & Number of entries \\
\hline Splicing sites & & GeneSplicer [15] & \\
\hline Splicing regulatory motifs & AEdb [8] & RegRNA 2.0 & 294 splicing motifs \\
\hline Polyadenylation Sites & & polya_svm [16] & \\
\hline Ribosome binding site & & RBSfinder [17] & \\
\hline Rho-independent terminator & & TransTermHP [18] & \\
\hline UTR Motifs & UTRsite [1] & PatSearch [22] & 48 UTRsite motifs \\
\hline AU-rich element & ARED [10] & RegRNA 2.0 & 5 ARE patterns \\
\hline RNA editing sites & & CURE [19] & \\
\hline Riboswitches & RiboSW models & RiboSW [21] & 12 riboswitches \\
\hline RNA elements & ERPIN profiles & ERPIN [23] & 11 RNA elements \\
\hline RNA cis-regulatory elements & Rfam CMs [4] & INFERNAL [24] & 209 Rfam cis-reg families \\
\hline Long stem & & einverted [30] & \\
\hline Known functional RNAs & fRNAdb [7] & BLAST [29] & 475,318 fRNAdb sequences \\
\hline miRNA target & miRBase [28] & miRanda [20] & 21,643 miRNA sequences \\
\hline ncRNA hybridization sites & NONCODE [11] & $\begin{array}{l}\text { BLAST [29], } \\
\text { RNAcofold [34] }\end{array}$ & 170,581 ncRNA sequences \\
\hline TRANSFAC motifs & TRANSFAC [14] & Match [25] & 2,171 transcription factor binding matrices \\
\hline User-defined motif & & RNAMotif [26] & \\
\hline Open Reading Frame & & RegRNA 2.0 & \\
\hline GC-content ratio & & RegRNA 2.0 & \\
\hline RNA accessibility & & RNAplfold [34] & \\
\hline
\end{tabular}

binding matrices of TRNASFAC, 294 sequence patterns of AEdb splicing regulatory motifs, 5 sequence patterns of ARED AU-rich elements, 475,318 fRNAdb sequences whose length are less than $500 \mathrm{nt}, 21,643$ miRNA sequences of miRBase and 170,581 ncRNA sequences of NONCODE collected for identifying different functional RNA motifs and sites.

\section{Development of identification procedures}

Numerous analytical approaches and data sources were integrated in RegRNA 2.0 (Table 1). GeneSplicer [15], polya_svm [16], RBSfinder [17], TransTermHP [18], CURE [19], RiboSW [21], and ERPIN [23], are incorporated for identifying splicing sites, polyadenylation sites, ribosome binding sites, Rho-independent terminator, C-to-U editing sites, riboswitches, and RNA elements, respectively. MATCH [25] is used with matrices collected in TRANSFAC [14] to provide the possibility to search for a variety of different transcription factor finding sites. PatSearch [22] and UTRsite models are integrated for indentifying UTR motifs. INFERNAL [24] and Rfam CMs are integrated for identifying cis-regulatory families. miRanda [20] and miRNA sequences of miRBase are integrated for identifying miRNA target sites. BLAST [29] and sequences of fRNAdb is integrated for finding similar functional RNA sequences. The einverted of EMBOSS package [30] is utilized for identifying long stems, which might be involved in mechanisms of gene regulatory processes [31-33]. For identifying putative RNA-RNA interaction sites, BLAST is used to find the complementary subsequence of input sequence against NONCODE database, and RNAcofold of Vienna RNA Package [34] is used to compute the free energy of hybridization sites. RNAMotif [26] is integrated for searching user-defined RNA motifs. In addition, RegRNA 2.0 is capable of predicting ORFs of the input RNA sequence. The default options are for resulting protein of at least 80 amino acids beginning with a start codon (AUG, GUG or UUG) and ending with a stop codon (UAA, UAG or UGA). The fully overlapped ORFs are not shown. Other related information, such as GC-content ratio and RNA accessibility, are also provided for the input RNA sequence. RNAplfold and RNAfold of Vienna RNA package [34] are used for predicting RNA accessibility and RNA secondary structure, respectively.

\section{User interface}

An integrated web-based system with user-friendly interface (Figure 2) was developed to facilitate user conveniently and comprehensively identifying functional RNA motifs and sites in an RNA sequence. User can submit a sequence by inputting a single sequence in FASTA format, or uploading a sequence file (Figure 2a), and the predictive results are presented via a graphical interface. User can decide which types of functional RNA motifs to be investigated by clicking the checkbox (Figure 2b). All parameters were set with default values, and user can alter the thresholds to fit their requirement. For instance, in predicting miRNA target sites, 


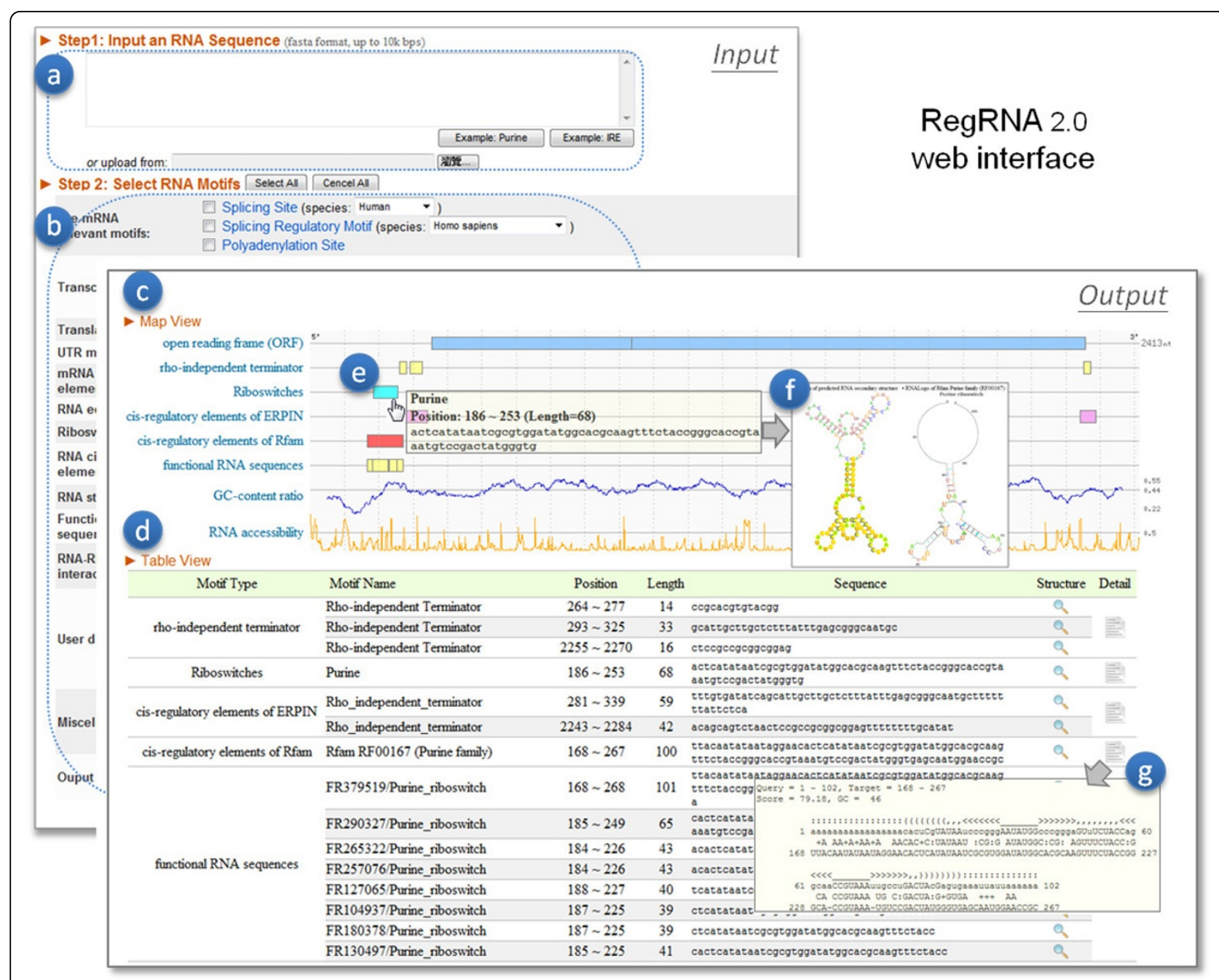

Figure 2 The RegRNA 2.0 web interface (a) input area (b) parameter setting (c) map view (d) table view (e) motif information (f) structure information $(g)$ details.

users can select the specie and adjust the minimum free energy (MFE) threshold and score threshold to filter miRNA targets of interest.

RegRNA 2.0 provides an intuitive graphical visualization (map view, Figure 2c) and summarized information table (table view, Figure 2d) for predictive results. The graphic location maps are created for intuitively displaying the positions of predictive motifs. The top-most graph shows the predictive ORFs, and the following graphs shows the predictive functional RNA motifs or sites. User can see the brief introduction of a predictive motif, such as the name, the start/end positions and the binding factors, by moving the cursor on it, and a pop-up description will be shown on the screen directly (Figure 2e). Further analysis and additional information of a predictive motif, such as the predictive secondary structure and the corresponding RNALogo [35] graph, can be observed by clicking on the motifs of interest (Figure 2f). The details of predictive results can be obtained in summarized information table (Figure 2g).

\section{Results}

\section{A case study of identification of purine riboswitch}

The purine riboswitch is used as a case study to demonstrate the capability of RegRNA 2.0. Purine riboswitches, which are found in the 5'UTR of mRNAs act as cis-acting genetic regulatory elements composed of a metaboliteresponsive aptamer domain in a specific secondary structure. It can regulate both transcription and translation by binding their corresponding targets. Additional file 1 illustrates a cartoon representation of the mechanism of genetic regulation by the guanine riboswitch [36]. In the presence high concentrations of guanine or hypoxanthine, ligand binding stabilizes the three-way junction structure, allowing 
the mRNA to form the terminator element (cyan). Without ligand binding, the 3'side of the P1 stem (green) and the 5 'side of the terminator are used to form an antiterminator element, allowing transcription to continue.

An RNA sequence with the accession number of EMBL, X83878, was used as an input for RegRNA 2.0. There exist a purine riboswitch and an operon of two genes, $B$. subtilis $x p t$ and $p u b X$, in X83878 according to the annotations of Rfam and EMBL database. The total length of X83878 is $2413 \mathrm{bps}$, and the location of purine riboswitch is from position 168 to 276 . The location of CDS regions of $x p t$ and $p u b X$ are from position 357 to 941 and from position 938 to 2254 , respectively.

Figure 3 shows the RegRNA 2.0 predictive results of the case study. The locations of two CDS regions were correctly predicted (Figure 3a), and the terminator of this operon was recognized by two RegRNA 2.0 identification procedures, TransTermHP and ERPIN (Figure $3 \mathrm{~b}$ ). The location of purine riboswitch was identified by three RegRNA 2.0 identification procedures, RiboSW, INFERNAL and BLAST fRNAdb (Figure 3c). A crucial RNA secondary structure, terminator, for purine riboswitch regulating gene activity was also predicted (Figure $3 \mathrm{~d}$ ), and the location of this terminator is close following the predictive purine riboswitch that corresponds to the mechanism of genetic regulation of the purine riboswitch [36]. In addition, the MFE secondary structure of predictive purine riboswitch regions shows the similar conformation to the RNALogo graph of Rfam purine family (Figure 3e). The results of case study show that RegRNA 2.0 is capable of identifying and displaying useful information in a given RNA sequence, and helpful for observing and deciphering RNA regulatory mechanisms.

\section{Discussions and conclusions}

RegRNA 2.0 facilitates user to identify functional RNA motifs and sites in an RNA sequence. As compared with our previously work, RegRNA [27], RegRNA 2.0 incorporates more data sources and analytical approaches (Table 2). RegRNA 2.0 enables user to identify more types of functional RNA motifs and sites including polyadenylation sites, ribosome binding sites, rho-independent terminator, AU-rich elements, RNA editing sites, RNA cis-regulatory elements, similar functional RNA sequences, non-coding RNA hybridization sites, long stems, open reading frames and related information of an RNA sequence. Additionally, RegRNA 2.0 provides further analysis, such as RNA secondary structure, RNA accessibility and RNALogo graph, for the predictive results, and display results with intuitive graphical visualization.

RegRNA 2.0 is an easy to use web server for comprehensively identifying regulatory RNA motifs and functional sites. It extends the widely used analysis platform, RegRNA [27], by taking more types of motifs and analytical approaches into consideration. RegRNA 2.0 is convenient to use programs without having to download the code and get the programs to run. Through its integrated userfriendly interface, user is capable of using various analytical approaches and observing results with graphical

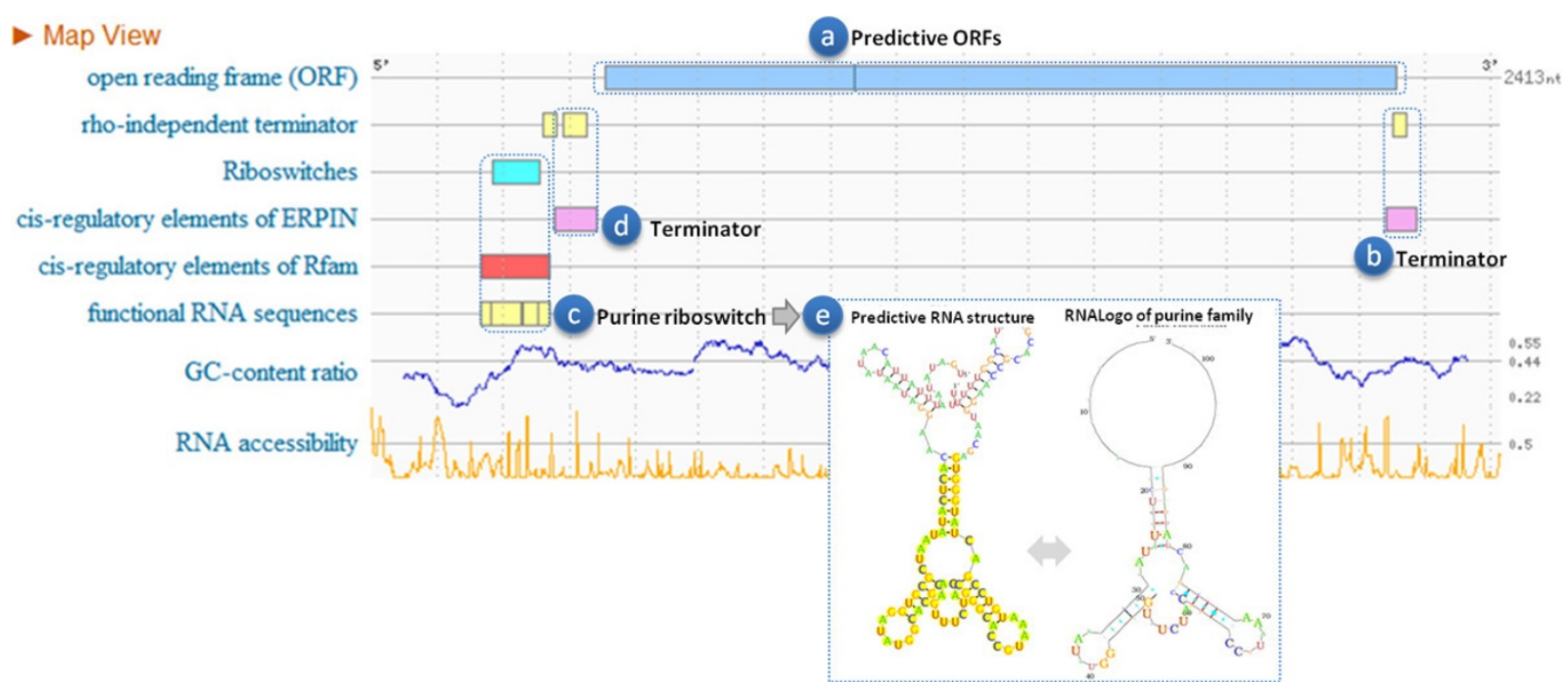

Figure 3 The RegRNA 2.0 predictive results of an input sequence, X83878, which is annotated with an operon of two genes and a purine riboswitch in EMBL and Rfam, respectively. (a) predictive ORFs (b) terminator (c) purine riboswitch (d) terminator (e) predictive structure and RNALogo of purine family. 
Table 2 A comparison between RegRNA 2.0 and RegRNA

\begin{tabular}{ccc}
\hline Features & RegRNA [27] & RegRNA 2.0 \\
\hline Polyadenylation sites & - & Yes (polya_svm) \\
Ribosome binding sites & - & Yes (RBSfinder) \\
Rho-independent terminator & - & Yes (TransTermHP) \\
RNA editing sites & - & Yes (CURE) \\
AU-rich elements & - & Yes (ARED) \\
RNA cis-elements & - & Yes (Rfam \& ERPIN) \\
similar functional RNAs & - & Yes (BLAST+fRNAdb) \\
ncRNA hybridization region & - & Yes (BLAST+RNAcofold+NONCODE) \\
Open reading frame & - & Yes (RegRNA 2.0) \\
Motif region structure & - & Yes (RNAfold) \\
RNALogo displaying & - & Yes (RNALogo) \\
GC-content Ratio & - & Yes (RegRNA 2.0) \\
RNA accessibility & - & Yes (RNAplfold) \\
DNA motifs & Yes (TRANSFAC 7.4) & Yes \& Updated (TRANSFC 2012.1) \\
Splicing regulatory motifs & Yes (AEDB 278 motifs) & Yes \& Updated (AEDB 294 motifs) \\
UTR motifs & Yes (UTRSite 40 motifs) & Yes \& Updated (UTRSite 48 motifs) \\
Riboswitches & Yes (RNAMotif) & Yes \& Updated (RiboSW \& Rfam) \\
miRNA target sites & Yes (744 miRNAs) & Yes \& Updated (miRBase 21,643 miRNAs) \\
Splicing sites & Yes & Yes (GeneSplicer) \\
Long stems & Yes & Yes (EMBOSS einverted) \\
User-defined Motifs & Yes & Yes (RNAMotif)
\end{tabular}

visualization conveniently. The platform will be enhanced by supporting input of multiple RNA sequences and providing conservation analysis in the future.

\section{Availability and requirements}

The RegRNA 2.0 system is freely available at http:// regrna2.mbc.nctu.edu.tw.

\section{Additional material}

Additional file 1: A cartoon representation of the mechanism of genetic regulation by the guanine riboswitch [36].

\section{Authors' contributions}

JTH and HDH conceived and supervised the study. THC, HYH, JBKH and SLW were responsible for the design, computational analyses, implementation of the system, and drafting the manuscript. All authors read and approved the final manuscript.

\section{Competing interests}

The authors declare that they have no competing interests.

\section{Acknowledgements}

The authors would like to thank the National Science Council of the Republic of China, No. NSC 101-2311-B-009-003-MY3 and NSC 100-2627-B009-002. This work was supported in part by the UST-UCSD International Center of Excellence in Advanced Bio-engineering sponsored by the Taiwan National Science Council I-RiCE Program under Grant Number: NSC 1012911-I-009-101, and Veterans General Hospitals and University System of Taiwan (VGHUST) Joint Research Program under Grant Number: VGHUST101G5-1-1. This work was also partially supported by MOE ATU.

\section{Declarations}

The authors approved the submission of this paper to BMC Bioinformatics for publication. The payment of publishing charges to BioMed Central for this article was supported by National Science Council of the Republic of China, No. NSC 101-2311-B-009-003-MY3 and NSC 100-2627-B-009-002. This publishing charge was supported in part by the UST-UCSD International Center of Excellence in Advanced Bio-engineering sponsored by the Taiwan National Science Council I-RiCE Program under Grant Number: NSC 1012911-I-009-101, and Veterans General Hospitals and University System of Taiwan (VGHUST) Joint Research Program under Grant Number: VGHUST101G5-1-1. This publishing charge is also partially supported by MOE ATU. This article has been published as part of BMC Bioinformatics Volume 14 Supplement 2, 2013: Selected articles from the Eleventh Asia Pacific Bioinformatics Conference (APBC 2013): Bioinformatics. The full contents of the supplement are available online at http://www.biomedcentral.com/ bmcbioinformatics/supplements/14/S2.

\section{Author details}

${ }^{1}$ Graduate Institute of Biomedical Informatics, Taipei Medical University, Taipei, Taiwan. ${ }^{2}$ Institute of Bioinformatics and Systems Biology, National Chiao Tung University, Hsin-Chu 300, Taiwan. ${ }^{3}$ Department of Biological Science and Technology, National Chiao Tung University, Hsin-Chu 300, Taiwan. ${ }^{4}$ Department of Obstetrics and Gynecology, Hsinchu Mackay Memorial Hospital, Hsinchu, Taiwan. ${ }^{5}$ Mackay Medicine, Nursing and Management College, Taipei, Taiwan. ${ }^{6}$ Department of Medicine, Mackay Medical College, New Taipei City, Taiwan. ${ }^{7}$ Department of Computer Science and Information Engineering, National Central University, Chung-Li 320, Taiwan. ${ }^{8}$ Institute of Systems Biology and Bioinformatics, National Central University, Chung-Li 320, Taiwan. ${ }^{9}$ Department of Biomedical informatics, Asia University, Wufeng 413, Taiwan.

Published: 21 January 2013

\section{References}

1. Mignone F, Grillo G, Licciulli F, lacono M, Liuni S, Kersey PJ, Duarte J, Saccone C, Pesole G: UTRdb and UTRsite: a collection of sequences and regulatory motifs of the untranslated regions of eukaryotic mRNAs. Nucleic acids research 2005, 33(Database):D141-146. 
2. Roth A, Breaker RR: The structural and functional diversity of metabolitebinding riboswitches. Annu Rev Biochem 2009, 78:305-334.

3. Montange RK, Batey RT: Riboswitches: emerging themes in RNA structure and function. Annu Rev Biophys 2008, 37:117-133.

4. Gardner PP, Daub J, Tate JG, Nawrocki EP, Kolbe DL, Lindgreen S, Wilkinson AC, Finn RD, Griffiths-Jones S, Eddy SR, et al: Rfam: updates to the RNA families database. Nucleic acids research 2009, 37(Database): D136-140

5. Griffiths-Jones S, Moxon S, Marshall M, Khanna A, Eddy SR, Bateman A: Rfam: annotating non-coding RNAs in complete genomes. Nucleic acids research 2005, 33(Database):D121-124.

6. Mituyama T, Yamada K, Hattori E, Okida H, Ono Y, Terai G, Yoshizawa A, Komori T, Asai K: The Functional RNA Database 3.0: databases to support mining and annotation of functional RNAs. Nucleic acids research 2009, 37(Database):D89-92.

7. Kin T, Yamada K, Terai G, Okida H, Yoshinari Y, Ono Y, Kojima A, Kimura Y, Komori T, Asai K: fRNAdb: a platform for mining/annotating functional RNA candidates from non-coding RNA sequences. Nucleic acids research 2007, 35(Database):D145-148.

8. Stamm S, Riethoven JJ, Le Texier V, Gopalakrishnan C, Kumanduri V, Tang Y, Barbosa-Morais NL, Thanaraj TA: ASD: a bioinformatics resource on alternative splicing. Nucleic acids research 2006, 34(Database):D46-55.

9. Bakheet T, Frevel M, Williams BR, Greer W, Khabar KS: ARED: human AUrich element-containing mRNA database reveals an unexpectedly diverse functional repertoire of encoded proteins. Nucleic acids research 2001, 29(1):246-254.

10. Bakheet T, Williams BR, Khabar KS: ARED 2.0: an update of AU-rich element mRNA database. Nucleic acids research 2003, 31(1):421-423.

11. He S, Liu C, Skogerbo G, Zhao H, Wang J, Liu T, Bai B, Zhao Y, Chen R: NONCODE v2.0: decoding the non-coding. Nucleic acids research 2008, 36(Database):D170-172.

12. Liu C, Bai B, Skogerbo G, Cai L, Deng W, Zhang Y, Bu D, Zhao Y, Chen R: NONCODE: an integrated knowledge database of non-coding RNAs. Nucleic acids research 2005, 33(Database):D112-115.

13. Griffiths-Jones S: miRBase: the microRNA sequence database. Methods Mol Biol 2006, 342:129-138

14. Matys V, Fricke E, Geffers R, Gossling E, Haubrock M, Hehl R, Hornischer K, Karas D, Kel AE, Kel-Margoulis OV, et al: TRANSFAC: transcriptional regulation, from patterns to profiles. Nucleic acids research 2003, 31(1):374-378.

15. Pertea M, Lin X, Salzberg SL: GeneSplicer: a new computational method for splice site prediction. Nucleic acids research 2001, 29(5):1185-1190

16. Cheng $Y$, Miura RM, Tian B: Prediction of mRNA polyadenylation sites by support vector machine. Bioinformatics 2006, 22(19):2320-2325.

17. Suzek BE, Ermolaeva MD, Schreiber M, Salzberg SL: A probabilistic method for identifying start codons in bacterial genomes. Bioinformatics 2001, 17(12):1123-1130.

18. Kingsford CL, Ayanbule K, Salzberg SL: Rapid, accurate, computational discovery of Rho-independent transcription terminators illuminates their relationship to DNA uptake. Genome Biol 2007, 8(2):R22.

19. DU P, Li Y: Prediction of C-to-U RNA editing sites in plant mitochondria using both biochemical and evolutionary information. J Theor Biol 2008, 253(3):579-586.

20. John B, Enright AJ, Aravin A, Tuschl T, Sander C, Marks DS: Human MicroRNA targets. PLoS Biol 2004, 2(11):e363.

21. Chang TH, Huang HD, Wu LC, Yeh CT, Liu BJ, Horng JT: Computational identification of riboswitches based on RNA conserved functional sequences and conformations. Rna 2009, 15(7):1426-1430.

22. Grillo G, Licciulli F, Liuni S, Sbisa E, Pesole G: PatSearch: A program for the detection of patterns and structural motifs in nucleotide sequences. Nucleic acids research 2003, 31(13):3608-3612.

23. Gautheret D, Lambert A: Direct RNA motif definition and identification from multiple sequence alignments using secondary structure profiles. J Mol Biol 2001, 313(5):1003-1011.

24. Nawrocki EP, Kolbe DL, Eddy SR: Infernal 1.0: inference of RNA alignments. Bioinformatics 2009, 25(10):1335-1337.

25. Kel AE, Gossling E, Reuter I, Cheremushkin E, Kel-Margoulis OV, Wingender E: MATCH: A tool for searching transcription factor binding sites in DNA sequences. Nucleic acids research 2003, 31(13):3576-3579.
26. Macke TJ, Ecker DJ, Gutell RR, Gautheret D, Case DA, Sampath R: RNAMotif, an RNA secondary structure definition and search algorithm. Nucleic acids research 2001, 29(22):4724-4735.

27. Huang $H Y$, Chien $\mathrm{CH}$, Jen $\mathrm{KH}$, Huang HD: RegRNA: an integrated web server for identifying regulatory RNA motifs and elements. Nucleic acids research 2006, 34(Web Server):W429-434.

28. Griffiths-Jones S, Saini HK, van Dongen S, Enright AJ: miRBase: tools for microRNA genomics. Nucleic acids research 2008, 36(Database):D154-158.

29. Altschul SF, Gish W, Miller W, Myers EW, Lipman DJ: Basic local alignment search tool. J Mol Biol 1990, 215(3):403-410.

30. Rice P, Longden I, Bleasby A: EMBOSS: the European Molecular Biology Open Software Suite. Trends Genet 2000, 16(6):276-277.

31. Gantier MP, Baugh JA, Donnelly SC: Nuclear transcription of long hairpin RNA triggers innate immune responses. I Interferon Cytokine Res 2007, 27(9):789-797.

32. Svoboda P, Di Cara A: Hairpin RNA: a secondary structure of primary importance. Cell Mol Life Sci 2006, 63(7-8):901-908.

33. Morse DP, Bass BL: Long RNA hairpins that contain inosine are present in Caenorhabditis elegans poly(A)+ RNA. Proc Natl Acad Sci USA 1999, 96(11):6048-6053.

34. Hofacker IL, Fontana W, Stadler PF, Bonhoeffer S, Tacker M, Schuster P: Fast Folding and Comparison of RNA Secondary Structures (The Vienna RNA Package). Monatshefte fur Chemie 1994, 125:167-188.

35. Chang TH, Horng JT, Huang HD: RNALogo: a new approach to display structural RNA alignment. Nucleic acids research 2008, 36(Web Server): W91-96.

36. Gilbert SD, Stoddard CD, Wise SJ, Batey RT: Thermodynamic and kinetic characterization of ligand binding to the purine riboswitch aptamer domain. J Mol Biol 2006, 359(3):754-768.

doi:10.1186/1471-2105-14-S2-S4

Cite this article as: Chang et al: An enhanced computational platform for investigating the roles of regulatory RNA and for identifying functional RNA motifs. BMC Bioinformatics 2013 14(Suppl 2):S4.

\section{Submit your next manuscript to BioMed Central and take full advantage of:}

- Convenient online submission

- Thorough peer review

- No space constraints or color figure charges

- Immediate publication on acceptance

- Inclusion in PubMed, CAS, Scopus and Google Scholar

- Research which is freely available for redistribution

Submit your manuscript at www.biomedcentral.com/submit
Ciomed Central 